\title{
Inpainting by Flexible Haar-Wavelet Shrinkage
}

\author{
R. H. Chan, S. Setzer and G. Steidl
}

March 20, 2008

\begin{abstract}
We present novel wavelet-based inpainting algorithms. Applying ideas from anisotropic regularization and diffusion our models can better handle degraded pixels at edges. We interpret our algorithms within the framework of forward-backward splitting methods in convex analysis and prove that the conditions to ensure their convergence are fulfilled. Numerical examples illustrate the good performance of our algorithms.
\end{abstract}

\section{Introduction}

The problem of inpainting occurs when part of the data in an image is missing. The task of inpainting is to recover the missing regions from the observed (sometimes noisy) incomplete data. The mathematical model for the image inpainting problem reads as follows: For convenience of notation we consider twodimensional images $\boldsymbol{u}$ defined on $\{1, \ldots, n\} \times\{1, \ldots, n\}$ and reshape them columnwise into a vector $u \in \mathbb{R}^{N}$ with $N=n^{2}$. Let the nonempty set $C \subset\{1, \ldots, N\}$ be the given region of the observed pixels. Then the observed incomplete image $f$ is

$$
f(j)= \begin{cases}u(j)+\varepsilon(j) & \text { if } j \in C, \\ \text { arbitrary } & \text { otherwise, }\end{cases}
$$

where $\varepsilon(j)$ denotes the noise. In the following, we denote by $P_{C}$ the diagonal matrix with diagonal entries 1 for indices in $C$ and 0 otherwise.

Initiated by [3], many useful techniques have been proposed to address this problem. In this paper we are mainly interested in wavelet-based inpainting methods. Such methods were, e.g., proposed in [6, 12]. However, often these methods let degraded pixels survive at sharp directed edges. A typical example is shown in Fig. 1. Here both the cubic spline interpolation and the wavelet-based method from [6] produce visible artefacts, in particular at the horizontal line. This was our motivation for considering more flexible wavelet-based methods.

We focus on the following general type of inpainting algorithms:

\section{Algorithm I (Exact Data)}

Initialization: $u_{0}$

For $r=0, \ldots$ iterate until convergence

i) Solve a restoration problem for the current image $u_{r}$ to obtain $\hat{u}_{r+1}$.

ii) Set

$$
u_{r+1}(j):= \begin{cases}f(j) & \text { if } j \in C, \\ \hat{u}_{r+1}(j) & \text { otherwise. }\end{cases}
$$



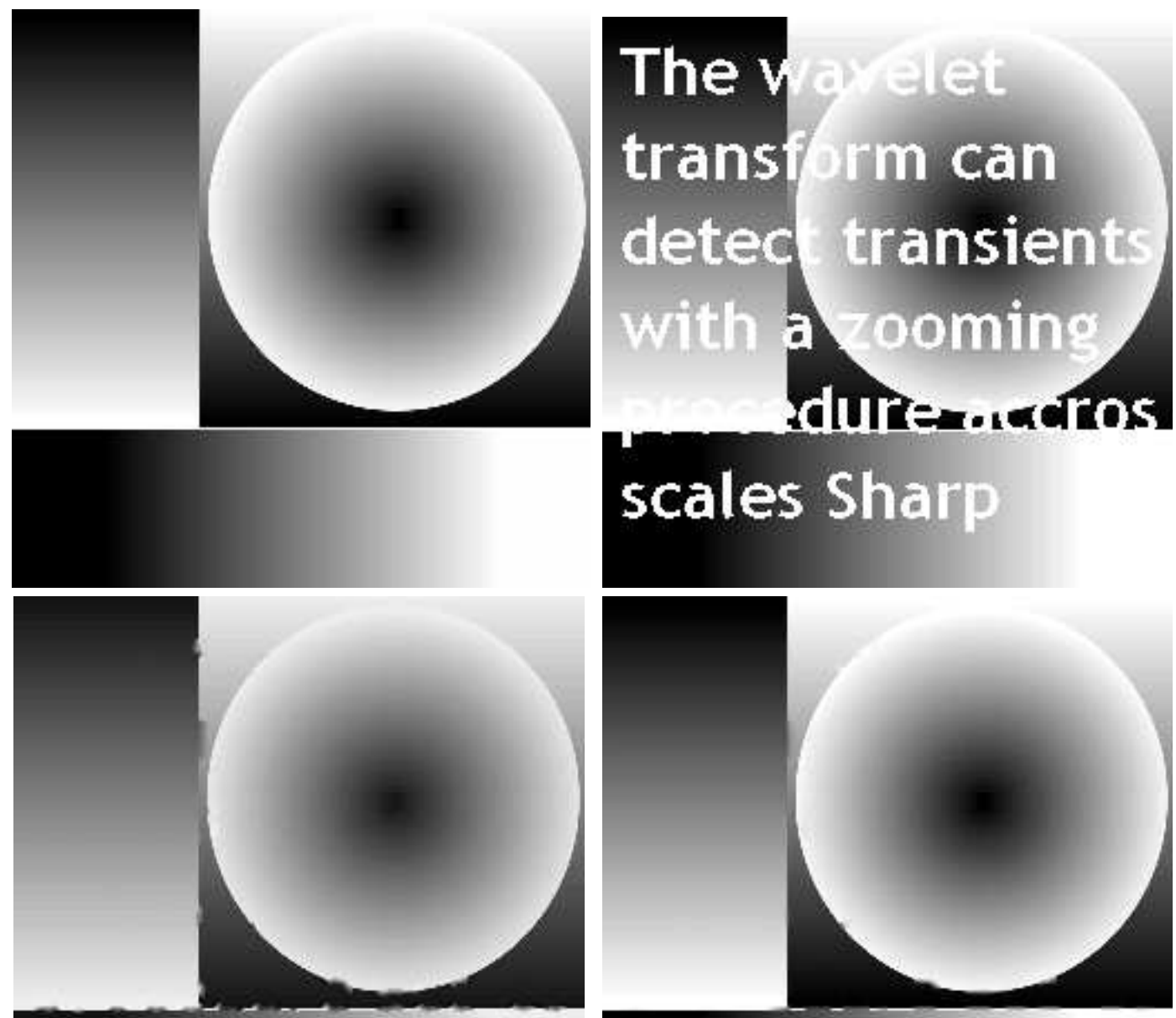

Figure 1: Top left: Original image. Top right: Degraded image. Bottom left: Cubic interpolation by the MATLAB routine 'griddata' $\left(\mathrm{PSNR}=29.39, \operatorname{err}_{2}=8.64, \operatorname{err}_{1}=0.79\right)$. Bottom right: Interpolation by the algorithm in [6] with $c=1$ and two levels ( $\mathrm{PSNR}=33.27$, $\operatorname{err}_{2}=5.53$, $\left.\operatorname{err}_{1}=0.46\right)$. The interpolated images have artefacts in particular at the horizontal line.

\section{Output: $u^{*}$}

\section{Algorithm II (Noisy data)}

Same as Algorithm I except that we have to apply step i) to the final iterate $u^{*}$ again. Output: $u^{\diamond}=\hat{u}^{*}$

Indeed, depending on the restoration method used in step i), many known inpainting algorithms are of this general type. In [6], the following wavelet-frame based denoising method was proposed for step i) of Algorithm I: Let $A \in \mathbb{R}^{M, N}, M \geq N$ denote a frame analysis operator of a Parseval frame, i.e., any $u \in \mathbb{R}^{N}$ can be written as $u=A^{\mathrm{T}} d$ and $A^{\mathrm{T}} A=I$. Further, 
let $\Lambda:=\operatorname{diag}(\lambda)$ be a diagonal matrix containing the components of the vector $\lambda:=\left(\lambda_{j}\right)_{j=1}^{M}$ as diagonal entries. Then the authors suggest to solve

$$
d_{r+1}=\underset{d \in \mathbb{R}^{M}}{\operatorname{argmin}} \frac{1}{2}\left\|A u_{r}-d\right\|_{2}^{2}+\|\Lambda d\|_{1} .
$$

Since the solution of $\operatorname{argmin}_{d} \frac{1}{2}\|c-d\|_{2}^{2}+\|\Lambda d\|_{1}$ is given by $\mathcal{T}_{\Lambda}(c)$ with the soft threshold operator $\mathcal{T}_{\Lambda}$ defined componentwise by

$$
T_{\lambda_{j}}\left(c_{j}\right)=\frac{1}{2}\left(\left(c_{j}-\lambda_{j}\right)+\left|c_{j}-\lambda_{j}\right|+\left(c_{j}+\lambda_{j}\right)-\left|c_{j}+\lambda_{j}\right|\right), \quad j=1, \ldots, M,
$$

see, e.g., [9], the restoration step i) becomes

$$
\hat{u}_{r+1}=A^{\mathrm{T}} \mathcal{T}_{\Lambda}\left(A u_{r}\right) .
$$

They proved that for noisy input data the iterates of Algorithm II with restoration step (1) converge to $u^{\diamond}=A^{\mathrm{T}} \hat{d}$, where $\hat{d}$ is the solution of

$$
\hat{d}=\underset{d \in \mathbb{R}^{M}}{\operatorname{argmin}} \frac{1}{2}\left\|P_{C} f-P_{C} A^{\mathrm{T}} d\right\|_{2}^{2}+\|\Lambda d\|_{1}+\frac{1}{2}\left\|\left(I-A A^{\mathrm{T}}\right) d\right\|_{2}^{2} .
$$

Indeed this algorithm is very similar to a method proposed in [12], where the authors solve

$$
\hat{d}=\underset{d \in \mathbb{R}^{M}}{\operatorname{argmin}} \frac{1}{2}\left\|P_{C} A^{\mathrm{T}} d-P_{C} f\right\|_{2}^{2}+\|\Lambda d\|_{1}
$$

by

$$
d_{r+1}=\mathcal{T}_{\Lambda}\left(d_{r}+A\left(P_{C} f-P_{C} A^{\mathrm{T}} d_{r}\right)\right)
$$

and set $u^{\diamond}=A^{\mathrm{T}} \hat{d}$. Obviously, for an orthogonal matrix $A$ the wavelet-based algorithms (2) and (3) coincide. However, for various non-orthogonal frame analysis matrices $A$, the numerical experiments in [6] indicate that the algorithm (2) performs better.

In [28], the method (3) was generalized in order to recover both the texture and the cartoon part of an image, see also [4]. To this end, the authors solve

$$
\underset{d_{t}, d_{n}}{\operatorname{argmin}} \frac{1}{2}\left\|P_{C}\left(A_{t}^{\mathrm{T}} d_{t}+A_{n}^{\mathrm{T}} d_{n}-f\right)\right\|_{2}^{2}+\lambda\left(\left\|d_{t}\right\|_{1}+\left\|d_{n}\right\|_{1}\right)+\gamma \operatorname{TV}\left(A_{n}^{\mathrm{T}} d_{n}\right),
$$

where $A_{n}$ denotes the discrete curvelet transform, $A_{t}$ the discrete cosine transform and $d_{t}$ and $d_{n}$ are the texture and cartoon components, respectively.

Beyond regularization techniques PDE-based approaches can be applied in the restoration step. In $[14,29]$ it was demonstrated that inpainting methods based on edge enhancing anisotropic diffusion appear to be superior to linear methods, e.g., spline interpolation methods, and nonlinear isotropic diffusion methods. Indeed these ideas were together with wavelet techniques the second ingredient for our algorithms. For other PDE-based methods we only refer to [7] and the references therein.

In this paper, we focus on inpainting by combining anisotropic regularization and diffusion methods with multilevel Haar wavelet filters. Our new methods increase the PSNR of various restored images significantly, e.g., by $3 \mathrm{~dB}$ for the image in Fig. 1 and avoid highly visible artefacts. Following the lines of [6], we have proved the convergence of our method by embedding it into the framework of forward-backward splitting algorithms. 
This paper is organized as follows: In Section 2, we briefly review anisotropic regularization and diffusion methods. Ideas from this section, in particular the application of a diffusion tensor, carry over to our wavelet setting. In Section 3, we present a new anisotropic Haarwavelet method for the inpainting problem. The convergence proof of our algorithm is given in Section 4. Finally, Section 5 contains numerical examples which demonstrate the excellent performance of our algorithm.

\section{Anisotropic Regularization and Diffusion}

In this section, we sketch the basic ideas from anisotropic diffusion and regularization methods that carry over to our wavelet setting. We prefer the more common continuous point of view in this section, while the rest of the paper deals with a discrete setting obtained by discretizing gradients with the help of wavelet filters. Anisotropic diffusion methods like edge enhancing or coherence enhancing diffusion were used for the directed denoising of images for a long time, see [26] and the references therein. Recently, anisotropic regularization methods became popular, e.g., for the restoration of polygonal shapes $[2,11,24]$ with sharp edges and corners.

Let us consider a single restoration step $r$ of our inpainting method which computes for a given continuous image $\tilde{f}:=u_{r}$ on a quadratic domain $\Omega$ the image $\hat{u}_{r+1}$. By $\circ$, we denote the Hadamard product (componentwise product) of matrices. From the variational point of view, one could restore the image by solving for an appropriate proper, lower semi-continuous (lsc), convex function $\Phi$ and an invertible matrix $V \in \mathbb{R}^{2,2}$ the problem

$$
\underset{u}{\operatorname{argmin}} \frac{1}{2}\|\tilde{f}-u\|_{L_{2}}^{2}+\lambda \int_{\Omega} \Phi\left(\left(V^{\mathrm{T}} \nabla u\right) \circ\left(V^{\mathrm{T}} \nabla u\right)\right) d x,
$$

where the function space of $u$ depends on the choice of $\Phi$. For $\Phi\left(x^{2}, y^{2}\right):=\sqrt{x^{2}+y^{2}}$ and $V:=I$, the functional in (4) is the Rudin-Osher-Fatemi (ROF) functional [21] and we consider the space $B V$ of functions of bounded variations. For $\Phi\left(x^{2}, y^{2}\right):=|x|+|y|$ and special rotation matrices $V$, the functional (4) was used for corner preserving denoising in [2, 24]. For $V=I$, minimization algorithms for this functional were considered, e.g., in [15]. If $\Phi$ is differentiable, then the Euler-Lagrange equation of (4) reads

$$
0=\tilde{f}-u+\lambda \nabla \cdot(D \nabla u)
$$

with

$$
D:=V\left(\begin{array}{cc}
2 \partial_{1} \Phi\left(\left(V^{\mathrm{T}} \nabla u\right) \circ\left(V^{\mathrm{T}} \nabla u\right)\right) & 0 \\
0 & 2 \partial_{2} \Phi\left(\left(V^{\mathrm{T}} \nabla u\right) \circ\left(V^{\mathrm{T}} \nabla u\right)\right)
\end{array}\right) V^{\mathrm{T}} .
$$

Here $\partial_{\nu}$ denotes the derivative with respect to the $\nu$-th variable. For example, we have for $\Phi\left(x^{2}, y^{2}\right):=\sqrt{x^{2}+y^{2}+\varepsilon^{2}}$ that $\partial_{1} \Phi\left(x^{2}, y^{2}\right)=\partial_{2} \Phi\left(x^{2}, y^{2}\right)=1 /\left(2 \sqrt{x^{2}+y^{2}+\varepsilon^{2}}\right)$ and for $\Phi\left(x^{2}, y^{2}\right):=\sqrt{x^{2}+\varepsilon^{2}}+\sqrt{y^{2}+\varepsilon^{2}}$ that $\partial_{1} \Phi\left(x^{2}, y^{2}\right)=1 /\left(2 \sqrt{x^{2}+\varepsilon^{2}}\right)$ and $\partial_{2} \Phi\left(x^{2}, y^{2}\right)=$ $1 /\left(2 \sqrt{y^{2}+\varepsilon^{2}}\right)$.

On the other hand, the so-called anisotropic edge enhancing diffusion (EED) acts via

$$
\begin{aligned}
\partial_{t} u & =\nabla \cdot(D \nabla u), \\
u(x, 0) & =\tilde{f}(x),
\end{aligned}
$$

with appropriate boundary conditions, mainly Neumann boundary conditions in image processing, and with the diffusion tensor

$$
D:=V\left(\begin{array}{cc}
g\left(\left|\nabla u_{\sigma}\right|\right) & 0 \\
0 & 1
\end{array}\right) V^{\mathrm{T}}, \quad V:=\left(\begin{array}{ll}
v & v^{\perp}
\end{array}\right), \quad v:=\frac{\nabla u_{\sigma}}{\left|\nabla u_{\sigma}\right|} .
$$


Here $u_{\sigma}=u * K_{\sigma}$ denotes the convolution of $u$ with the Gaussian of standard deviation $\sigma$ and $g$ is a decreasing nonnegative function. In applications, the function

$$
g(|s|)= \begin{cases}1-e^{-\frac{3.31488}{(s / \alpha)^{8}}} & s>0 \\ 1 & s=0\end{cases}
$$

introduced by Weickert in [26] has shown a good performance.

A relation to regularization methods can be seen as follows: If we use instead of (8) the matrices (6), then (5) can be considered as a semidiscretization of (7) with an implicit Euler step of time step size $\lambda$. The following wavelet methods are related to explicit time discretizations so that we can only achieve approximations of the corresponding regularization method. For further investigations in this direction see [23]. Note that according to [26] we will call a method anisotropic if the diagonal matrix in the diffusion tensor contains different nonzero diagonal entries. In this sense, the ROF method is an isotropic one.

\section{Anisotropic Haar-Wavelet Shrinkage}

In this section, we return to our discrete setting from the beginning of the paper. Let $h_{0}:=\frac{1}{2}\left[\begin{array}{ll}1 & 1\end{array}\right]$ and $h_{1}:=\frac{1}{2}\left[\begin{array}{ll}1 & -1\end{array}\right]$ be the filters of the Haar wavelet. For convenience of notation, we use periodic boundary conditions and denote by $H_{0} \in \mathbb{R}^{n, n}$ and $H_{1} \in \mathbb{R}^{n, n}$ the corresponding circulant matrices. A remark concerning Neumann boundary conditions can be found at the end of this section. The following remark shows the link between the continuous considerations in the previous section and our discrete setting. Basically we consider discretizations of continuous images on a regular grid and approximate the partial derivatives by special differences related to our Haar wavelet filters.

Remark 3.1 i) Discretizing a periodic smooth function $u$ on $[0,1)^{2}$ at the grid $\{(j, k) / h$ : $j, k=0, \ldots, n-1\}$ and setting $\boldsymbol{u}:=\left(u_{j, k}\right)_{j, k=0}^{n-1}=\left(u\left(\frac{j}{h}, \frac{k}{h}\right)\right)_{j, k=0}^{n-1}$, we see by using the twodimensional Taylor expansion that

$$
\frac{u_{j+1, k+1}-u_{j, k+1}+u_{j+1, k}-u_{j, k}}{2 h}=\partial_{x} u\left(j+\frac{h}{2}, k+\frac{h}{2}\right)+\mathcal{O}\left(h^{2}\right),
$$

i.e., the left-hand side is a consistent discretization of $\partial_{x} u$. In matrix-vector notation this yields the following approximation of $\nabla u$ :

$$
2\left(\begin{array}{c}
H_{1} \boldsymbol{u} H_{0}^{T} \\
H_{0} \boldsymbol{u} H_{1}^{T}
\end{array}\right)=-h\left(\begin{array}{c}
\left(\partial_{x} u\left(j+\frac{h}{2}, k+\frac{h}{2}\right)\right)_{j, k=0}^{n-1} \\
\left(\partial_{y} u\left(j+\frac{h}{2}, k+\frac{h}{2}\right)\right)_{j, k=0}^{n-1}
\end{array}\right)+\mathcal{O}\left(h^{2}\right) .
$$

Reshaping $\boldsymbol{u}$ columnwise into a vector $u$ and using that $R \boldsymbol{u} S^{T}=(S \otimes R) u$, the left-hand side of (9) becomes $2\left(\begin{array}{c}H_{0} \otimes H_{1} \\ H_{1} \otimes H_{0}\end{array}\right)$ u. For digital images one sets $h:=1$.

ii) In [27], an $\ell_{2}$-stable, conditionally consistent, so-called 'locally semi-analytic scheme' (LSAS) for the numerical solution of the EED equation (7) was developed. It involves a sophisticated spatial discretization and an explicit Euler scheme as temporal discretization. With respect to our notation the iterative LSAS scheme computes at every time step with time step size $\tau$ based on the old iterate $u_{\text {old }}$ the new one $u_{\text {new }}$ by the following steps: 
1. $\left(\begin{array}{c}c_{00} \\ c_{01} \\ c_{10} \\ c_{11}\end{array}\right):=\underbrace{\left(\begin{array}{c}H_{0} \otimes H_{0} \\ H_{0} \otimes H_{1} \\ H_{1} \otimes H_{0} \\ H_{1} \otimes H_{1}\end{array}\right)}_{A} u_{\text {old }}$,

2. $\left(\begin{array}{c}d_{01} \\ d_{10}\end{array}\right):=V\left(\begin{array}{cc}e^{-4 \tau g\left(\left|\nabla u_{\text {old }, \sigma}\right|\right)} & 0 \\ 0 & e^{-4 \tau}\end{array}\right) V^{T}\left(\begin{array}{c}c_{01} \\ c_{10}\end{array}\right), \quad \begin{array}{ll}d_{00} & :=c_{00}, \\ d_{11} & :=e^{-4 \tau\left(g\left(\left|\nabla u_{o l d, \sigma}\right|\right)+1\right)} c_{11},\end{array}$

3. $u_{\text {new }}:=A^{T}\left(d_{00}^{T}, d_{01}^{T}, d_{10}^{T}, d_{11}^{T}\right)^{T}$,

where

$$
\nabla u_{\text {old }, \sigma}:=2\left(\begin{array}{c}
H_{0} \otimes H_{1} \\
H_{1} \otimes H_{0}
\end{array}\right)\left(u_{\text {old }} * K_{\sigma}\right)
$$

and $V$ is chosen in accordance to (8) as $V:=\left(\begin{array}{rr}c & -s \\ s & c\end{array}\right)$ with $c:=\operatorname{diag}\left(\left(\left(H_{0} \otimes H_{1}\right) u_{o l d, \sigma}\right) / w\right)$, $s:=\operatorname{diag}\left(\left(\left(H_{1} \otimes H_{0}\right) u_{\text {old }, \sigma}\right) / w\right), w:=\sqrt{\left(\left(H_{0} \otimes H_{1}\right) u_{\text {old }, \sigma}\right)^{2}+\left(\left(H_{1} \otimes H_{0}\right) u_{\text {old }, \sigma}\right)^{2}}$ and componentwise quotients $\left(\left(H_{0} \otimes H_{1}\right) u_{\text {old }, \sigma}\right) / w$ and squares $\left(\left(H_{0} \otimes H_{1}\right) u_{\text {old }, \sigma}\right)^{2}$ of vectors.

We consider the undecimated discrete Haar wavelet transform up to level $m$. For $k=$ $1, \ldots, m$, let $H_{\nu}^{(k)} \in \mathbb{R}^{n, n}, \nu \in\{0,1\}$ be the circulant matrix corresponding to the filter $h_{\nu}^{(k)}=\frac{1}{2}(1, \underbrace{0, \ldots, 0}_{2^{k-1}-1},(-1)^{\nu})$ with $2^{k-1}-1$ inserted zeros between the filter coefficients. Further, we set

$$
\left(\begin{array}{c}
H_{00}^{(k)} \\
H^{(k)} \\
H_{11}^{(k)}
\end{array}\right):=\left(\begin{array}{c}
H_{00}^{(k)} \\
H_{10}^{(k)} \\
H_{01}^{(k)} \\
H_{11}^{(k)}
\end{array}\right)=\left(\begin{array}{c}
H_{0}^{(k)} \otimes H_{0}^{(k)} \\
H_{0}^{(k)} \otimes H_{1}^{(k)} \\
H_{1}^{(k)} \otimes H_{0}^{(k)} \\
H_{1}^{(k)} \otimes H_{1}^{(k)}
\end{array}\right) \prod_{l=1}^{k-1}\left(H_{0}^{(l)} \otimes H_{0}^{(l)}\right) .
$$

Then the matrix

$$
A=\left(\begin{array}{c}
H_{00}^{(m)} \\
H^{(1)} \\
\vdots \\
H^{(m)} \\
H_{11}^{(1)} \\
\vdots \\
H_{11}^{(m)}
\end{array}\right) \in \mathbb{R}^{(3 m+1) N, N}
$$

satisfies $A^{\mathrm{T}} A=I$ while $A A^{\mathrm{T}} \neq I$. Let $V^{(k)}$ be orthogonal matrices and let $\Lambda^{(k)}:=$ $\operatorname{diag}\left(\lambda_{j}^{(k)}\right)_{j=1}^{2 N}, \Lambda_{11}^{(k)}:=\operatorname{diag}\left(\lambda_{11, j}^{(k)}\right)_{j=1}^{N}, k=1, \ldots, m$ be diagonal matrices with nonnegative entries. For $p \in[1,2]$, we consider the minimization problem

$$
\underset{u \in \mathbb{R}^{N}}{\operatorname{argmin}} \frac{1}{2}\|f-u\|_{2}^{2}+\frac{1}{p} \sum_{k=1}^{m}\left\|\Lambda^{(k)}\left(V^{(k)}\right)^{\mathrm{T}} H^{(k)} u\right\|_{p}^{p}+\frac{1}{p} \sum_{k=1}^{m}\left\|\Lambda_{11}^{(k)} H_{11}^{(k)} u\right\|_{p}^{p} .
$$


In our numerical examples, we will only use $p=1$ and $p=2$. Since $A^{\mathrm{T}} A=I$, this is equivalent to

$$
\underset{u \in \mathbb{R}^{N}}{\operatorname{argmin}} \frac{1}{2}\|A f-A u\|_{2}^{2}+\frac{1}{p} \sum_{k=1}^{m}\left\|\Lambda^{(k)}\left(V^{(k)}\right)^{\mathrm{T}} H^{(k)} u\right\|_{p}^{p},+\frac{1}{p} \sum_{k=1}^{m}\left\|\Lambda_{11}^{(k)} H_{11}^{(k)} u\right\|_{p}^{p} .
$$

Using the notation

$$
c:=A f=\left(c_{00}^{(m)}, c^{(1)}, \ldots, c^{(m)}, c_{11}^{(1)}, \ldots, c_{11}^{(m)}\right)^{\mathrm{T}}, \quad d:=A u
$$

this can be rewritten as

$$
\underset{d \in \mathbb{R}^{(3 m+1) N}}{\operatorname{argmin}} \frac{1}{2}\|c-d\|_{2}^{2}+\frac{1}{p} \sum_{k=1}^{m}\left\|\Lambda^{(k)}\left(V^{(k)}\right)^{\mathrm{T}} d^{(k)}\right\|_{p}^{p}+\frac{1}{p} \sum_{k=1}^{m}\left\|\Lambda_{11}^{(k)} d_{11}^{(k)}\right\|_{p}^{p} \quad \text { s.t. } \quad d \in \mathcal{R}(A) .
$$

Note that $d \in \mathcal{R}(A)$ is equivalent to $\left(I-A A^{\mathrm{T}}\right) d=0$, i.e., the orthogonal projection of $d$ onto the kernel of $A^{\mathrm{T}}$ has to be 0 . In other words, if $\hat{d}$ is a solution of this problem, then $A A^{\mathrm{T}} \hat{d}$ is just the orthogonal projection of $\hat{d}$ onto $\mathcal{R}(A)$. We will not solve this minimization problem in step i) of our inpainting algorithm, but the following one which is obtained by neglecting the constraint:

$$
\underset{d \in \mathbb{R}^{(3 m+1) N}}{\operatorname{argmin}} \frac{1}{2}\|c-d\|_{2}^{2}+J_{\Lambda, p}(d),
$$

where

$$
J_{\Lambda, p}(d):=\frac{1}{p} \sum_{k=1}^{m}\left\|\Lambda^{(k)}\left(V^{(k)}\right)^{\mathrm{T}} d^{(k)}\right\|_{p}^{p}+\frac{1}{p} \sum_{k=1}^{m}\left\|\Lambda_{11}^{(k)} d_{11}^{(k)}\right\|_{p}^{p} .
$$

This functional can be decoupled as

$$
\begin{aligned}
& \frac{1}{2}\left\|c_{00}^{(m)}-d_{00}^{(m)}\right\|_{2}^{2}+\sum_{k=1}^{m}\left(\frac{1}{2}\left\|c^{(k)}-d^{(k)}\right\|_{2}^{2}+\frac{1}{p}\left\|\Lambda^{(k)}\left(V^{(k)}\right)^{\mathrm{T}} d^{(k)}\right\|_{p}^{p}\right) \\
& +\sum_{k=1}^{m}\left(\frac{1}{2}\left\|c_{11}^{(k)}-d_{11}^{(k)}\right\|_{2}^{2}+\frac{1}{p}\left\|\Lambda_{11}^{(k)} d_{11}^{(k)}\right\|_{p}^{p}\right) .
\end{aligned}
$$

Now the three parts of the functional can be minimized separately which leads to the following solution.

Lemma 3.2 The minimizer $\hat{d}$ of the functional (12) is given by

$$
\begin{aligned}
\hat{d}_{00}^{(m)} & =c_{00}^{(m)} \\
\hat{d}^{(k)} & =V^{(k)} \mathcal{T}_{\Lambda^{(k)}, p}\left(\left(V^{(k)}\right)^{T} c^{(k)}\right), \quad k=1, \ldots, m \\
\hat{d}_{11}^{(k)} & =\mathcal{T}_{\Lambda_{11}^{(k)}, p}\left(c_{11}^{(k)}\right), \quad k=1, \ldots, m
\end{aligned}
$$

with the following shrinkage procedures $\mathcal{T}_{\text {.,. : }}$

i) the soft shrinkage $\mathcal{T}_{\Lambda, 1}$ for $p=1$,

ii) $\mathcal{T}_{\Lambda, p}(y)=F_{\Lambda, p}^{-1}(y)$, where $F_{\Lambda, p}$ is the injective mapping

$$
F_{\Lambda, p}(x)=x+\Lambda^{p}\left(\operatorname{sgn}(x) \circ|x|^{p-1}\right)
$$

for $p \in(1,2)$, 
iii) $\mathcal{T}_{\Lambda, 2}(y):=\left(I+\Lambda^{2}\right)^{-1} y$ for $p=2$.

Moreover, we have for $p \in(1,2]$ that

$$
\left|\mathcal{T}_{\Lambda, p}(y)\right|^{p} \geq\left(I+\Lambda^{p}\right)^{-p}|y|^{p}-1 .
$$

Proof: Since the matrices $V^{(k)}$ are orthogonal, we obtain immediately assertion i).

In the following, we restrict our attention to the central functional, i.e., to $\hat{d}^{(k)}$. For $p \in(1,2]$, the functional is differentiable and the minimizer has to fulfill

$$
\begin{aligned}
0 & =\hat{d}^{(k)}-c^{(k)}+V^{(k)} \Lambda^{(k)}\left(\operatorname{sgn}\left(\Lambda^{(k)}\left(V^{(k)}\right)^{\mathrm{T}} \hat{d}^{(k)}\right) \circ\left|\Lambda^{(k)}\left(V^{(k)}\right)^{\mathrm{T}} \hat{d}^{(k)}\right|^{p-1}\right) \\
\left(V^{(k)}\right)^{\mathrm{T}} c^{(k)} & =\left(V^{(k)}\right)^{\mathrm{T}} \hat{d}^{(k)}+\left(\Lambda^{(k)}\right)^{p} \operatorname{sgn}\left(\left(V^{(k)}\right)^{\mathrm{T}} \hat{d}^{(k)}\right) \circ\left|\left(V^{(k)}\right)^{\mathrm{T}} \hat{d}^{(k)}\right|^{p-1}
\end{aligned}
$$

Then $x=\left(V^{(k)}\right)^{\mathrm{T}} \hat{d}^{(k)}$ is the solution of $\left(V^{(k)}\right)^{\mathrm{T}} c^{(k)}=F_{\Lambda^{(k)}, p}(x)$ and $\hat{d}^{(k)}=V^{(k)} x$. In particular, we have for $p=2$ that $x=\left(I+\Lambda^{2}\right)^{-1}\left(V^{(k)}\right)^{\mathrm{T}} c^{(k)}$.

We prove the last assertion (14) componentwise. For $x, y \in \mathbb{R}$ and $\lambda \in \mathbb{R}_{\geq 0}$ the equation $y=x+\lambda^{p} \operatorname{sgn}(x)|x|^{p-1}$ implies that

$$
|y|=|x|+\lambda^{p}|x|^{p-1} .
$$

Then, we see for $|x| \geq 1$ and $p \in(1,2]$ that $|y| \leq|x|+\lambda^{p}|x|$ and consequently $|x| \geq(1+$ $\left.\lambda^{p}\right)^{-1}|y|$. For $|x|<1$, we have that $|y| \leq|x|^{p-1}+\lambda^{p}|x|^{p-1}$ so that $1>|x|^{p-1} \geq\left(1+\lambda^{p}\right)^{-1}|y|$. Thus, $1>\left(1+\lambda^{p}\right)^{-p}|y|^{p}$ and $|x|^{p} \geq 0>\left(1+\lambda^{p}\right)^{-p}|y|^{p}-1$.

Let us denote the whole shrinkage procedure by $\hat{d}=\mathcal{T}_{\Lambda, p} c$. Finally, we can compute the denoised image $u$ of $f$ by $u=A^{\mathrm{T}} \hat{d}$. With this denoising procedure our inpainting algorithm reads as follows:

\section{Algorithm I.1 (Exact Data)}

Initilization: $u_{0}$

For $r=0, \ldots$ iterate until convergence

i) Compute $\hat{u}_{r+1}=A^{\mathrm{T}} \mathcal{T}_{\Lambda, p}\left(A u_{r}\right)$ with $\mathcal{T}_{\Lambda, p}$ defined by Lemma 3.2 .

ii) Set

$$
u_{r+1}(j):= \begin{cases}f(j) & \text { if } j \in C \\ \hat{u}_{r+1}(j) & \text { otherwise. }\end{cases}
$$

Output: $u^{*}$

\section{Algorithm II.1 (Noisy data)}

Same as Algorithm I except that we have to apply step i) to the final iterate $u^{*}$ again. Output: $u^{\diamond}=\hat{u}^{*}$

The set

$$
\mathcal{C}:=\left\{g \in \mathbb{R}^{N}: g(j)=f(j), \forall j \in C\right\} .
$$

is nonempty, closed, convex so that its indicator function $\iota_{\mathcal{C}}$ is a proper lsc convex function. Thus, step ii) of the inpainting procedure also reads

$$
u_{r+1}=\underset{u \in \mathbb{R}^{N}}{\operatorname{argmin}} \frac{1}{2}\left\|\hat{u}_{r+1}-u\right\|_{2}^{2}+\iota_{\mathcal{C}}(u) .
$$


Thus, the whole algorithm can be rewritten in the form

$$
\begin{aligned}
& d_{r+1}=\underset{d \in \mathbb{R}^{(3 m+1) N}}{\operatorname{argmin}} \frac{1}{2}\left\|A u_{r}-d\right\|_{2}^{2}+J_{\Lambda, p}(d), \\
& u_{r+1}=\underset{u \in \mathbb{R}^{N}}{\operatorname{argmin}} \frac{1}{2}\left\|A^{\mathrm{T}} d_{r+1}-u\right\|_{2}^{2}+\iota_{\mathcal{C}}(u),
\end{aligned}
$$

where $J_{\Lambda, p}(d)$ is defined in (11).

Remark 3.3 (Neumann boundary conditions)

If we assume mirrored boundaries, we have to replace the circulant matrices $H_{\nu}, \nu=0,1$ by the Toeplitz matrices $H_{\nu} \in \mathbb{R}^{n+1, n+2}$ corresponding to the filters $h_{\nu}$. Let $\tilde{H}_{\nu} \in \mathbb{R}^{n+1, n}$ denote the matrices obtained from $H_{\nu}^{T}$ by canceling their first and last row. Then we have that

$$
H_{0}^{T} H_{0}+H_{1}^{T} H_{1}=\left(\begin{array}{ccc}
\frac{1}{2} & & \\
& I & \\
& & \frac{1}{2}
\end{array}\right), \quad \text { and } \quad \tilde{H}_{0} H_{0}+\tilde{H}_{1} H_{1}=I .
$$

Consider one decomposition level $m=1$. For higher levels we have to incorporate the corresponding zeros into the filters and to mirror the boundaries according to the filter length. Let $\tilde{\mathbf{f}}$ denote the image obtained from $\mathbf{f}$ by mirroring the boundaries and let $A, \tilde{A}$ be defined as in (10) but with the new Toeplitz matrices $H_{\nu}, \tilde{H}_{\nu}, \nu=0,1$. Then we solve instead of (12) the minimization problem

$$
\hat{d}_{r}=\underset{d}{\operatorname{argmin}}\left\{\frac{1}{2}\left\|A \tilde{u}_{r}-d\right\|_{2}^{2}+\frac{1}{p}\left\|\Lambda V^{T} d\right\|_{p}^{p}+\frac{1}{p}\left\|\Lambda_{11} d_{11}\right\|_{p}^{p}\right\}
$$

and set $\hat{u}_{r}:=\tilde{A} \hat{d}_{r}$.

\section{Convergence Considerations}

Following [6], we show the convergence of our inpainting algorithm by identifying it as a forward-backward splitting algorithm to minimize the sum of two operators. There exists a vast literature on forward-backward splitting algorithms and related fixed point iterations, see Remark 4.2 below. In this paper, we need only the following setting in the Hilbert space $\mathbb{R}^{N}$ with the Euclidian norm.

For any proper, convex, lower semi-continuous (lsc) function $\varphi$ the proximal operator is defined by

$$
\operatorname{prox}_{\varphi}(x)=\underset{y \in \mathbb{R}^{N}}{\operatorname{argmin}}\left\{\frac{1}{2}\|x-y\|_{2}^{2}+\varphi(y)\right\}
$$

and its envelope by

$$
{ }^{1} \varphi(x)=\min _{y \in \mathbb{R}^{N}}\left\{\frac{1}{2}\|x-y\|_{2}^{2}+\varphi(y)\right\} .
$$

By [1, Theorem 5.2], the function ${ }^{1} \varphi$ is convex and differentiable, and its gradient is

$$
\nabla\left({ }^{1} \varphi\right)(x)=x-\operatorname{prox}_{\varphi}(x) .
$$


Lemma 4.1 [6] Let $F_{1}: \mathbb{R}^{N} \rightarrow \mathbb{R} \cup\{+\infty\}$ be a proper, convex, lsc function and let $F_{2}$ : $\mathbb{R}^{N} \rightarrow \mathbb{R} \cup\{+\infty\}$ be a proper, convex, differentiable function with a Lipschitz continuous gradient with Lipschitz constant $<2$. Assume that

$$
\underset{u \in \mathbb{R}^{N}}{\operatorname{argmin}}\left\{F_{1}(u)+F_{2}(u)\right\}
$$

has a solution. Then, for any initial guess $u_{0}$, the so-called proximal forward-backward splitting

$$
u_{r+1}=\operatorname{prox}_{F_{1}}\left(u_{r}-\nabla F_{2}\left(u_{r}\right)\right)
$$

converges to a minimizer of the functional $F_{1}+F_{2}$.

The iteration (18) is a special case of a more general class of algorithms which we briefly outline in the following remark.

Remark 4.2 For subdifferentiable functions $F_{1}, F_{2}: \mathcal{H} \rightarrow \mathbb{R} \cup\{+\infty\}$ on a Hilbert space $\mathcal{H}$ we have that

$$
\hat{u}=\underset{u \in \mathcal{H}}{\operatorname{argmin}}\left\{F_{1}(u)+F_{2}(u)\right\} \quad \Leftrightarrow \quad 0 \in \partial\left(F_{1}+F_{2}\right)(\hat{u}) .
$$

Under certain conditions on $F_{1}$ and $F_{2}$ this is equivalent to $0 \in \partial F_{1}(\hat{u})+\partial F_{2}(\hat{u})$. If $\partial F_{1}, \partial F_{2}$ are maximal monotone operators, Lions and Mercier [17] and independently Passty [20] suggested to solve the inclusion on the right-hand side by the splitting iteration

$$
\hat{u}=\left(I+c \partial F_{1}\right)^{-1}\left(I-c \partial F_{2}\right) \hat{u} .
$$

Under certain conditions on $\partial F_{2}$ and the step size $c$ it was proved that the Picard iteration of (19) converges weakly to a minimizer $\hat{u}$, see, e.g., [13, 25]. Meanwhile there exist various generalizations of this algorithm as those in [8].

Since in our special problem $F_{1}$ is proper, convex, lsc and $F_{2}$ is differentiable, we have that $\left(I+\partial F_{1}\right)^{-1}=\operatorname{prox}_{F_{1}}$ and $\partial F_{2}=\nabla F_{2}$, so that (19) with $c=1$ coincides with (18).

We now return to Algorithm I.1. For our problem, we set $F_{1}:=\iota_{C}$ and $F_{2}:=\left({ }^{1} J_{\Lambda, p}\right)(A \cdot)$. Then we obtain

$$
F_{2}(u)=\min _{d \in \mathbb{R}^{(3 m+1) N}}\left\{\frac{1}{2}\|A u-d\|_{2}^{2}+J_{\Lambda, p}(d)\right\}
$$

so that

$$
\begin{aligned}
F_{1}(u)+F_{2}(u) & =\iota_{C}(u)+\min _{d \in \mathbb{R}^{(3 m+1) N}}\left\{\frac{1}{2}\|A u-d\|_{2}^{2}+J_{\Lambda, p}(d)\right\} \\
& =\iota_{C}(u)+\frac{1}{2}\left\|A u-\mathcal{T}_{\Lambda, p}(A u)\right\|_{2}^{2}+J_{\Lambda, p}\left(\mathcal{T}_{\Lambda, p}(A u)\right) .
\end{aligned}
$$

Further, we obtain by (17) that $F_{2}$ is differentiable with

$$
\nabla F_{2}(u)=\nabla\left({ }^{1} J_{\Lambda, p} \circ A\right)(u)=A^{\mathrm{T}}\left(A u-\operatorname{prox}_{J_{\Lambda, p}}(A u)\right) .
$$


Now the forward-backward splitting (18) becomes

$$
\begin{aligned}
u_{r+1} & =\operatorname{prox}_{F_{1}}\left(u_{r}-\nabla F_{2}\left(u_{r}\right)\right) \\
& =\underset{u \in \mathbb{R}^{N}}{\operatorname{argmin}}\left\{\frac{1}{2}\left\|u_{r}-\nabla F_{2}\left(u_{r}\right)-u\right\|_{2}^{2}+\iota_{C}(u)\right\} \\
& =\underset{u \in \mathbb{R}^{N}}{\operatorname{argmin}}\left\{\frac{1}{2}\left\|u_{r}-A^{\mathrm{T}}\left(A u_{r}-\operatorname{prox}_{J_{\Lambda, p}}\left(A u_{r}\right)\right)-u\right\|_{2}^{2}+\iota_{C}(u)\right\} \\
& =\underset{u \in \mathbb{R}^{N}}{\operatorname{argmin}}\left\{\frac{1}{2}\left\|A^{\mathrm{T}} \operatorname{prox}_{J_{\Lambda, p}}\left(A u_{r}\right)-u\right\|_{2}^{2}+\iota_{C}(u)\right\} .
\end{aligned}
$$

By (15) and (16) this coincides with the sequence produced by our Algorithm I.1.

Next, we show that $F_{1}+F_{2}$ in (20) is coercive.

Lemma 4.3 The functional $F_{1}+F_{2}$ in (20) is coercive.

Proof: By (20) we obtain

$$
F_{1}(u)+F_{2}(u)=\iota_{C}(u)+\frac{1}{2}\left\|A u-\mathcal{T}_{\Lambda, p}(A u)\right\|_{2}^{2}+J_{\Lambda, p}\left(\mathcal{T}_{\Lambda, p}(A u)\right) \geq J_{\Lambda, p}\left(\mathcal{T}_{\Lambda, p}(A u)\right) .
$$

Let $A u:=\left((A u)_{00}^{(m)},(A u)^{(1)}, \ldots,(A u)^{(m)},(A u)_{11}^{(1)}, \ldots,(A u)_{11}^{(m)}\right)^{\mathrm{T}}$. Then we see by $(12)$ and (13) that

$$
J_{\Lambda, p}\left(\mathcal{T}_{\Lambda, p}(A u)\right)=\frac{1}{p} \sum_{k=1}^{m}\left\|\Lambda^{(k)} \mathcal{T}_{\Lambda^{(k), p}}\left(\left(V^{(k)}\right)^{\mathrm{T}}(A u)^{(k)}\right)\right\|_{p}^{p}+\frac{1}{p} \sum_{k=1}^{m}\left\|\Lambda_{11}^{(k)} \mathcal{T}_{\Lambda_{11}^{(k)}, p}\left((A u)_{11}^{(k)}\right)\right\|_{p}^{p}
$$

Now we have by (14) and by definition of the soft shrinkage function that

$$
\frac{1}{p}\left(\lambda_{j}^{(k)}\right)^{p}\left|\mathcal{T}_{\lambda_{j}^{(k)}, p}(y)\right|^{p} \geq \begin{cases}\lambda_{j}^{(k)}|y|-\left(\lambda_{j}^{(k)}\right)^{2} & \text { for } p=1, \\ \frac{1}{p}\left(\frac{\lambda_{j}^{(k)}}{1+\left(\lambda_{j}^{(k)}\right)^{p}}\right)^{p}|y|^{p}-\frac{1}{p}\left(\lambda_{j}^{(k)}\right)^{p} & \text { for } p \in(1,2] .\end{cases}
$$

Thus, setting

$$
\kappa_{1}:=\frac{1}{p} \min \left\{\left(\frac{\lambda_{j}^{(k)}}{1+\left(\lambda_{j}^{(k)}\right)^{p}}\right)^{p},\left(\frac{\lambda_{11, i}^{(k)}}{1+\left(\lambda_{11, i}^{(k)}\right)^{p}}\right)^{p}: j=1, \ldots, 2 N ; i=1, \ldots, N ; k=1, \ldots, m\right\}
$$

and

$$
\kappa_{2}:= \begin{cases}\sum_{k=1}^{m}\left(\sum_{j=1}^{2 N}\left(\lambda_{j}^{(k)}\right)^{2}+\sum_{i=1}^{N}\left(\lambda_{11, j}^{(k)}\right)^{2}\right) & \text { for } p=1, \\ \frac{1}{p} \sum_{k=1}^{m}\left(\sum_{j=1}^{2 N}\left(\lambda_{j}^{(k)}\right)^{p}+\sum_{i=1}^{N}\left(\lambda_{11, j}^{(k)}\right)^{p}\right) & \text { for } p \in(1,2]\end{cases}
$$

and applying that $\|x\|_{p} \geq\|x\|_{2}$ for $p \in[1,2]$ we get

$$
\begin{aligned}
J_{\Lambda, p}\left(\mathcal{T}_{\Lambda, p}(A u)\right) & \geq \kappa_{1}\left(\sum_{k=1}^{m}\left\|\left(V^{(k)}\right)^{\mathrm{T}}(A u)^{(k)}\right\|_{p}^{p}+\sum_{k=1}^{m}\left\|(A u)_{11}^{(k)}\right\|_{p}^{p}\right)-\kappa_{2} \\
& \geq \kappa_{1}\left(\sum_{k=1}^{m}\left\|(A u)^{(k)}\right\|_{2}^{p}+\sum_{k=1}^{m}\left\|(A u)_{11}^{(k)}\right\|_{2}^{p}\right)-\kappa_{2} .
\end{aligned}
$$


Using the notation $A_{0}:=H_{00}^{(m)}, A_{1}:=\left(\left(H^{(1)}\right)^{\mathrm{T}}, \ldots,\left(H^{(m)}\right)^{\mathrm{T}},\left(H^{(1)}\right)_{11}^{\mathrm{T}}, \ldots,\left(H^{(m)}\right)_{11}^{\mathrm{T}}\right)^{\mathrm{T}}$, this can be rewritten as

$$
J_{\Lambda, p}\left(\mathcal{T}_{\Lambda, p}(A u)\right) \geq \kappa_{1}\left\|A_{1} u\right\|_{2}^{p}-\kappa_{2} .
$$

By Lemma 4.4 below, the matrix $A_{0}^{\mathrm{T}} A_{0}$ has the simple eigenvalue 1 with a corresponding normed eigenvector $\tilde{u}=\frac{1}{\sqrt{N}} \mathbf{1}_{N}$. Since $A^{\mathrm{T}} A=I$, it follows that $A_{1}^{\mathrm{T}} A_{1}$ has the simple eigenvalue 0 and that the kernel of $A_{1}^{\mathrm{T}} A_{1}$ is spanned by $\tilde{u}$. Now we obtain for the orthogonal decomposition $u=v+a \tilde{u}$ that $|a| \geq\|u\|_{2}-\|v\|_{2}$ and

$$
\left\|A_{1} u\right\|_{2}^{2}=\left\|A_{1} v\right\|_{2}^{2} \geq \eta_{2}\|v\|_{2}^{2}
$$

where $\eta_{2}>0$ is the second smallest eigenvalue of $A_{1}^{\mathrm{T}} A_{1}$. Now we fix a constant $c \in\left(\frac{1}{\sqrt{N}+1}, 1\right)$ and consider two cases:

1. For $\|v\|_{2} \geq c\|u\|_{2}$, we conclude by (24) and (25) that

$$
F_{1}(u)+F_{2}(u) \geq \kappa_{1}\left\|A_{1} u\right\|_{2}^{p}-\kappa_{2} \geq \kappa_{1}{\sqrt{\eta_{2}}}^{p}\|v\|_{2}^{p}-\kappa_{2} \geq \kappa_{1}{\sqrt{\eta_{2}}}^{p} c^{p}\|u\|_{2}^{p}-\kappa_{2} .
$$

2. For $\|v\|_{2}<c\|u\|_{2}$ it holds that $|a|>(1-c)\|u\|_{2}$. Hence, we have for any $i_{0} \in C$ that

$$
\left|u_{i_{0}}\right|=\left|v_{i_{0}}+a \tilde{u}_{i_{0}}\right| \geq|a|\left|\tilde{u}_{i_{0}}\right|-\left|v_{i_{0}}\right|>(1-c)\|u\|_{2}\left|\tilde{u}_{i_{0}}\right|-c\|u\|_{2}=\|u\|_{2} \frac{1-c(1+\sqrt{N})}{\sqrt{N}} .
$$

Thus, we see for $\|u\|_{2}$ large enough that $\left|u_{i_{0}}\right|>\left|f_{i_{0}}\right|$ and consequently $F_{1}(u)+F_{2}(u) \geq \iota_{C}(u)=$ $+\infty$.

Lemma 4.4 The matrix $A_{0}^{T} A_{0}$ has 1 as a simple eigenvalue with corresponding eigenvector $\tilde{u}=\frac{1}{\sqrt{N}} \mathbf{1}_{N}$.

Proof: Using multiplication rules for tensor products we obtain that

$$
A_{0}^{\mathrm{T}} A_{0}=B^{\mathrm{T}} B \otimes B^{\mathrm{T}} B, \quad B:=\prod_{l=1}^{m} H_{0}^{(l)}=\frac{1}{2^{m}} \operatorname{circ}\left([\underbrace{1 \ldots 1}_{2^{m}} 0 \ldots 0]\right) .
$$

By [10], the circulant matrix $B$ has eigenvectors $\frac{1}{\sqrt{n}}\left(e^{-\frac{2 \pi i j k}{n}}\right)_{j=0}^{n}$ and eigenvalues $\beta_{0}=1$ and

$$
\left|\beta_{k}\right|=\left|\frac{1}{2^{m}} \sum_{j=0}^{2^{m}-1} e^{-\frac{2 \pi i j k}{n}}\right|=\frac{1}{2^{m}} \frac{\left|1-e^{-\frac{2 \pi i j 2^{m}}{n}}\right|}{\left|1-e^{-\frac{2 \pi i j}{n}}\right|}=\frac{1}{2^{m}} \prod_{p=1}^{m}\left|1+e^{-\frac{2 \pi i j 2^{m-p}}{n}}\right|<1,
$$

$k=1, \ldots, n-1$. The last inequality holds true because $\left|1+e^{-\frac{2 \pi i j 2 m-p}{n}}\right| \leq 2$ for $p=1, \ldots, m$ with strict inequality for $p=m$.

In summary, we obtain the following convergence result.

Theorem 4.5 The sequence $\left\{u_{r}\right\}_{r=0}^{\infty}$ produced by Algorithm I.1 converges for any start image $u_{0}$ and $p \in[1,2]$ to a minimizer of the functional $F_{1}+F_{2}$ in $(20)$. 
Proof: By (22), the sequence produced by our Algorithm I.1 coincides with the sequence generated by the forward-backward splitting algorithm (18). Now the assertion follows since the functional $F_{1}+F_{2}$ in (20) fulfills the convergence assumptions of Lemma 4.1: The functions $F_{1}$ and $F_{2}$ are proper, convex and lsc. By Lemma 4.3 the functional $F_{1}+F_{2}$ is coercive so that there exists at least one minimizer of the functional. Finally, since $\|A\|_{2}=1$ and $I-\operatorname{prox}_{J_{\Lambda, p}}$ is nonexpansive, it is easy to check as in [6] that $F_{2}$ has a gradient with Lipschitz constant 1.

With respect to Remark 4.2 we notice that for our setting $\left(I+\partial F_{1}\right)^{-1}\left(I-\partial F_{2}\right)$ is an averaged operator, i.e., the strictly convex combination of the identity operator and a nonexpansive mapping. Alternatively to Lemma 4.1 one could also use convergence results for Picard iterations of averaged operators, see [5, 16, 18, 19, 22].

Remark 4.6 Numerical experiments indicate that Algorithm I.1 converges linearly. However, we have not proved this so far. In [25, Proposition 1 (d)], Tseng gives a sufficient condition for linear convergence. Unfortunately, it cannot be applied here since neither $\partial F_{1}$ nor $\nabla F_{2}$ are strongly monotone.

\section{$5 \quad$ Numerical Examples}

Finally, we present some numerical examples, in particular, we compare our algorithm with the algorithm in [6] without thresholding of the smoothest coefficients. Since the results for noisy data with a small amount of noise are similar as those for exact data, we restrict our attention to exact input data.

All programs were written in MATLAB. We have always assumed Neumann boundary conditions. We have used the stopping criterion for the iterations $\left\|u_{r+1}-u_{r}\right\|_{2} /\left\|u_{r+1}\right\|_{2} \leq$ $5 \cdot 10^{-5}$. We compare the weighted $\ell_{1}$-error $\operatorname{err}_{1}:=\|u-f\|_{1} / N$, the weighted $\ell_{2}$-error $\operatorname{err}_{2}:=$ $\|u-f\|_{2} / \sqrt{N}$, and the PSNR $:=20 \cdot \log _{10}\left(255 / \operatorname{err}_{2}\right)$. The parameters were chosen with respect to the "best" PSNR.

We compare the following algorithms:

(A) the wavelet-based algorithm in [6] with the filters $h_{0}:=\frac{1}{4}\left[\begin{array}{lll}1 & 2 & 1\end{array}\right], h_{1}:=\frac{\sqrt{2}}{4}\left[\begin{array}{lll}1 & 0 & -1\end{array}\right]$ and $h_{2}:=\frac{1}{4}\left[\begin{array}{lll}-1 & 2-1\end{array}\right]$ and soft shrinkage of the high-pass coefficients at level $k$ with the thresholds $c / \sqrt{2^{k}}$.

(B) the Algorithm I.1 with our Haar wavelet filters, $p=1, V^{(k)}=I$ and soft shrinkage with threshold $\lambda_{j}^{(k)}:=\lambda / \sqrt{2^{k}}$ and $\lambda_{11, j}^{(k)}:=\lambda_{11} / \sqrt{2^{k}}$ at level $k$.

(C) same algorithm as in (B) except that we use matrices $V^{(k)}$ inspired by the LSAS scheme explained in Remark 3.1ii): we convolve an appropriate guess $\tilde{f}$ of the original function with the Gaussian of standard derivation $\sigma$ to obtain $\tilde{f}_{\sigma}$. Then, at level $k$, we set $V^{(k)}:=\left(\begin{array}{rr}c^{(k)} & -s^{(k)} \\ s^{(k)} & c^{(k)}\end{array}\right)$ with $c^{(k)}:=\operatorname{diag}\left(H_{01}^{(k)} \tilde{f}_{\sigma} / w\right), s^{(k)}:=\operatorname{diag}\left(H_{10}^{(k)} \tilde{f}_{\sigma} / w\right), w^{(k)}:=$ $\sqrt{\left(H_{01}^{(k)} \tilde{f}_{\sigma}\right)^{2}+\left(H_{10}^{(k)} \tilde{f}_{\sigma}\right)^{2}}$, i.e., we use the same matrices $V^{(k)}$ in each iteration step $r$.

(D) the Algorithm I.1 with $p=2$ and the following setting inspired by the LSAS scheme for 
EED in Remark 3.1 ii): we define $V^{(k)}$ as in $(\mathrm{C})$. In the shrinkage step we use

$$
\begin{array}{ll}
\left(I+\left(\Lambda^{(k)}\right)^{2}\right)^{-1} & :=\left(\begin{array}{cc}
\operatorname{diag}\left(e^{-4 \tau g\left(w^{(k)}\right)}\right) & 0 \\
0 & \operatorname{diag}\left(e^{-4 \tau} 1_{N}\right)
\end{array}\right), \\
\left(I+\left(\Lambda_{11}^{(k)}\right)^{2}\right)^{-1} & :=\operatorname{diag}\left(e^{-4 \tau\left(g\left(w^{(k)}\right)+1\right)}\right)
\end{array}
$$

with the vector $1_{N}$ of $N$ ones. We still use the same matrices $V^{(k)}, \Lambda^{(k)}$ and $\Lambda_{11}^{(k)}$ in each iteration step $r$.

(E) same algorithm as in (D) except that we do not freeze $V^{(k)}$ and the shrinkage matrices at the beginning of the algorithm with respect to $\tilde{f}_{\sigma}$ but compute them in each iteration step $r$ with respect to the actual iterate $u_{r}$. Note that we have not proved the convergence for this algorithm. If we would work only with one level of Haar wavelet decomposition $m=1$, then the restoration step can be considered as one time step of an iterative EED scheme discretized by LSAS.

In our first example we start with the image at the top right of Fig. 1 which we also use as initial guess $u_{0}$. Alternatively, one could use the image in Fig. 1 bottom left generated by the MATLAB cubic interpolation procedure 'griddata' as initial guess. This leads to qualitatively similar results but with a fewer number of iterations. However, we have used this cubic interpolation in the algorithms $\mathrm{C}$ and $\mathrm{D}$ for $\tilde{f}$. Detailed results are given in the tables below. Here 'iter' denotes the number of iterations. The corresponding images for the decomposition level 2 are depicted in Fig. 2 and at the bottom right of Fig. 1. The algoritms described in $\mathrm{B}-\mathrm{E}$ perform much better than the algorithm in A. The PSNR improves by approximately $3 \mathrm{~dB}$ if we use algorithms $\mathrm{B}-\mathrm{D}$ and by approximately $5 \mathrm{~dB}$ for algorithm E. The algorithms B-E considerably reduce the artefacts at the horizontal line. However, the algorithms B and $\mathrm{C}$ introduce some errors at the boundary of the circle. These artefacts do not appear if we apply the algorithms D and E. In general, the PSNR cannot be substantially improved by choosing a higher decomposition level than $m=2$.

\begin{tabular}{c|c|r|r|r|r} 
level & $c$ & PSNR & err $_{2}$ & err $_{1}$ & iter \\
\hline 4 & 1.0 & 32.93 & 5.49 & 0.54 & 307 \\
3 & 1.0 & 33.29 & 5.51 & 0.48 & 307 \\
2 & 1.0 & $\mathbf{3 3 . 2 7}$ & 5.53 & 0.46 & 358 \\
1 & 1.6 & 32.50 & 6.04 & 0.50 & 461
\end{tabular}

\begin{tabular}{c|c|r|r|r|r|r} 
level & $\lambda$ & $\lambda_{11}$ & PSNR & err $_{2}$ & err $_{1}$ & iter \\
\hline 4 & 1 & 8 & 34.84 & 4.61 & 0.36 & 272 \\
3 & 1 & 10 & 35.52 & 4.27 & 0.29 & 235 \\
2 & 1 & 100 & $\mathbf{3 6 . 4 2}$ & 3.84 & 0.27 & 278 \\
1 & 1 & 100 & 35.89 & 4.09 & 0.28 & 814
\end{tabular}

Results of the inpainting algorithms A (left) and B (right).

\begin{tabular}{c|c|c|r|r|r|r|r} 
level & $\sigma$ & $\lambda$ & $\lambda_{11}$ & PSNR & err $_{2}$ & err $_{1}$ & iter \\
\hline 4 & 4 & 1 & 8 & 35.43 & 4.31 & 0.36 & 244 \\
3 & 4 & 1 & 10 & 35.97 & 4.05 & 0.30 & 223 \\
2 & 4 & 1 & 100 & $\mathbf{3 6 . 6 0}$ & 3.76 & 0.26 & 269 \\
1 & 4 & 1 & 100 & 36.03 & 4.02 & 0.26 & 811
\end{tabular}

Results of the inpainting algorithms C.

\begin{tabular}{c|c|c|c|r|r|c|cc|c|c|c|c|c|c|c} 
level & $\sigma$ & $\tau$ & $\alpha$ & PSNR & $\operatorname{err}_{2}$ & err $_{1}$ & iter & level & $\sigma$ & $\tau$ & $\alpha$ & PSNR & err $_{2}$ & err $_{1}$ & iter \\
\hline 4 & 4 & 1 & 2 & 35.19 & 4.43 & 0.47 & 73 & & 4 & 1 & 2 & 35.91 & 4.07 & 0.44 & 78 \\
3 & 4 & 1 & 2 & 36.08 & 4.00 & 0.36 & 79 & 3 & 4 & 1 & 2 & 37.47 & 3.40 & 0.29 & 88 \\
2 & 4 & 1 & 2 & $\mathbf{3 6 . 7 9}$ & 3.68 & 0.29 & 106 & 2 & 4 & 1 & 2 & $\mathbf{3 8 . 5 8}$ & 2.99 & 0.23 & 123 \\
1 & 4 & 1 & 2 & 36.83 & 3.83 & 0.28 & 208 & 1 & 4 & 1 & 2 & 37.99 & 3.21 & 0.24 & 215
\end{tabular}

Results of the inpainting algorithms D (left) and E (right). 

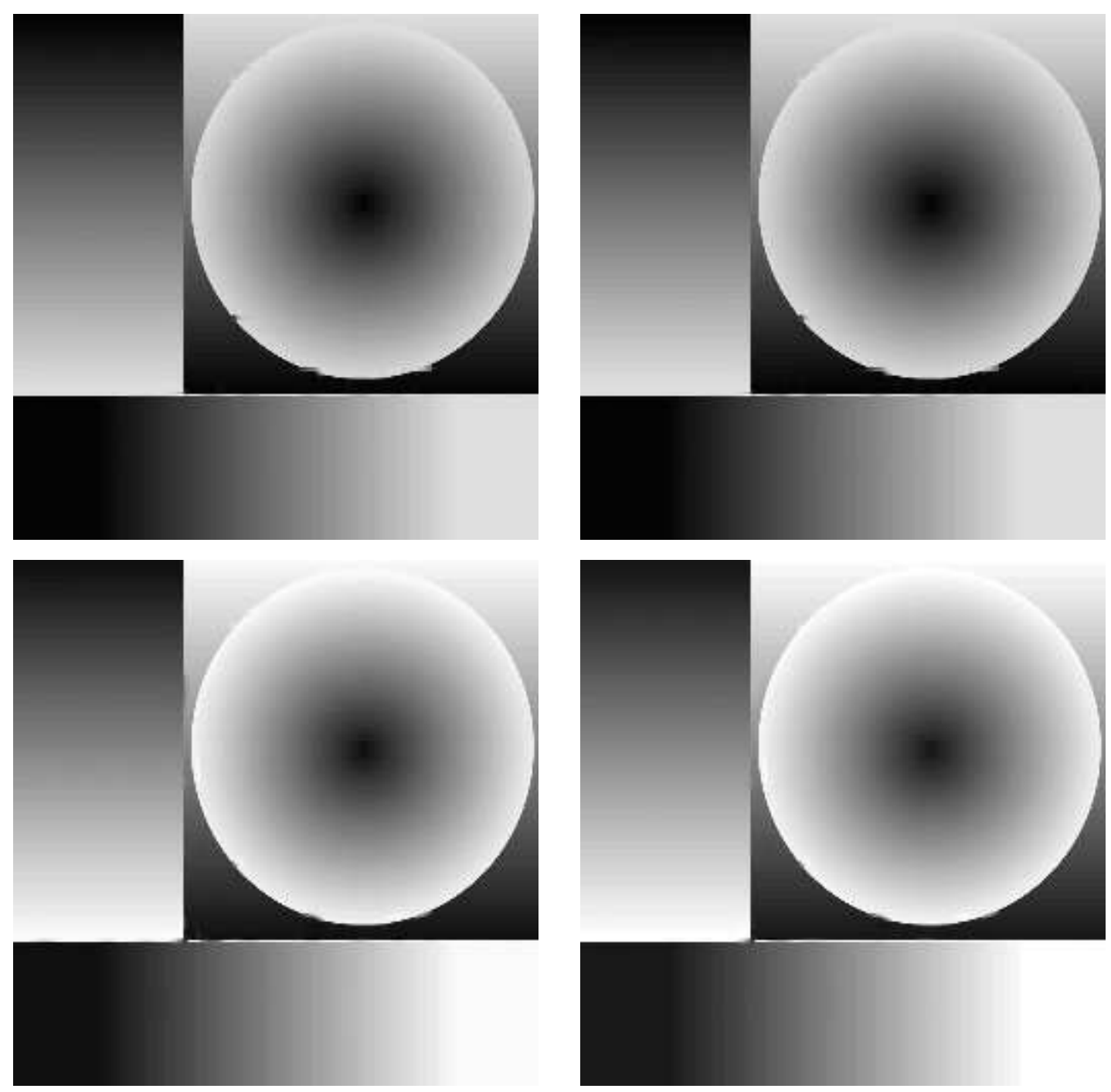

Figure 2: Applications of the algorithms in B-E with decomposition level 2. Top left: algorithm $\mathrm{B},\left(\mathrm{PSNR}=36.42, \mathrm{err}_{2}=3.84, \mathrm{err}_{1}=0.27\right)$. Top right: algorithm $\mathrm{C},(\mathrm{PSNR}=36.60$, $\left.\operatorname{err}_{2}=3.36, \operatorname{err}_{1}=0.26\right)$. Bottom left: algorithm D, $\left(\mathrm{PSNR}=36.79, \operatorname{err}_{2}=3.68, \operatorname{err}_{1}=0.29\right)$. Bottom right: algorithm $\mathrm{E},\left(\mathrm{PSNR}=38.58, \mathrm{err}_{2}=2.99\right.$, $\left.\operatorname{err}_{1}=0.23\right)$. All algorithms reduce the artefacts at the straight lines. However, the images at the top contain similar errors at the boundary of the circle. The images at the bottom have the best quality.

In our second example we interpolate the image on the right-hand side of Fig. 3. Again, we use this image as initial guess and its cubic interpolation as $\tilde{f}$ in the algorithms $\mathrm{C}$ and D. This cubic interpolation is depicted at the top left of Fig. 4 and contains hard artefacts at the windows on the left-hand side. The results for our algorithms with two decomposition levels look as follows:

- Algorithm A with $c=1.0$ : PSNR=31.61, $\operatorname{err}_{2}=6.69, \operatorname{err}_{1}=1.36$.

- Algorithm B with $\lambda=0.5$ and $\lambda_{11}=8$ : PSNR=34.08, $\operatorname{err}_{2}=5.03, \operatorname{err}_{1}=0.93$. 
- Algorithm C with $\sigma=0.5, \lambda=0.5$ and $\lambda_{11}=8$ : $\mathrm{PSNR}=33.98, \operatorname{err}_{2}=5.09, \operatorname{err}_{1}=0.97$.

- Algorithm D with $\sigma=1, \tau=1$ and $\alpha=2$ : PSNR=31.56, $\operatorname{err}_{2}=6.73, \operatorname{err}_{1}=1.27$.

- Algorithm E with $\sigma=1, \tau=1$ and $\alpha=2$ : PSNR=31.36, $\operatorname{err}_{2}=6.89, \operatorname{err}_{1}=1.26$.

The algorithms $\mathrm{B}$ and $\mathrm{C}$ perform best. The PSNR is approximately $2 \mathrm{~dB}$ higher than in the other three algorithms. While the algorithms A, D and E produce similar artefacts especially at the windows, these errors do not appear if we apply the algorithms B and C. This is illustrated in Fig. 4 and in the zoomed images in Fig. 5.
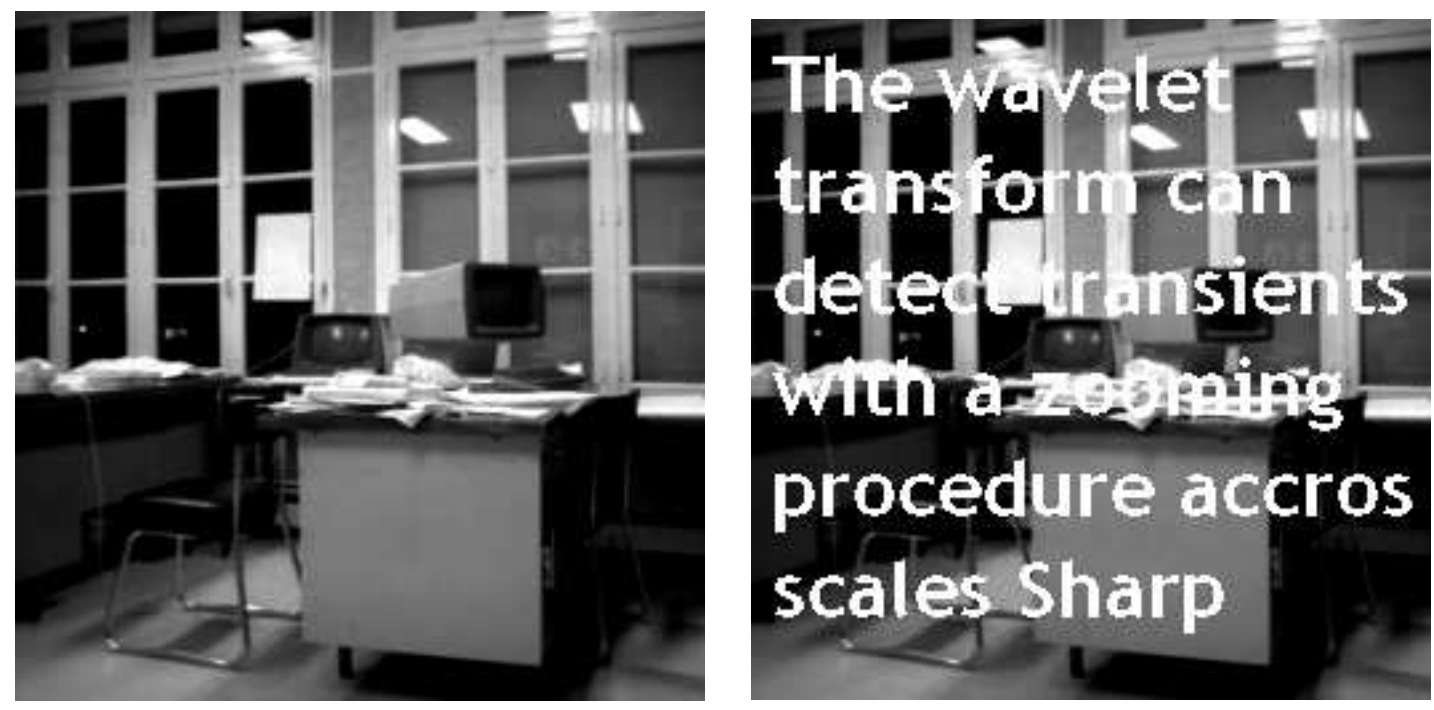

Figure 3: Original image of the second example and its degraded version.

In our third example, we consider the image at the top left of Fig. 6. For this image cubic interpolation yields very good results $(\mathrm{PSNR}=33.62)$, see top right of Fig. 6. Starting with this image as an initial guess and using small parameters $\left(c=\lambda=\lambda_{11}=0.05\right)$, we can achieve a PSNRs of around 33.8 by applying algorithms A-C. Visual differences to the image obtained by cubic interpolation are hard to find. For the algorithms D and $\mathrm{E}$ with the original image as initial guess, two decomposition levels and parameters $\sigma=1, \tau=1$ and $\alpha=10$ we obtain the PSNR $=34.25$, $\operatorname{err}_{2}=4.93, \operatorname{err}_{1}=0.98$ after 86 iterations and the PSNR $=34.21$, $\operatorname{err}_{2}=4.96$, $\operatorname{err}_{1}=1.00$ after 249 iterations, respectively. As shown at the bottom of Fig. 6 there are visual differences at long edges.

\section{References}

[1] J.-P. Aubin, Optima and Equilibria: An Introduction to Nonlinear Analysis, Springer, 2nd edition, Berlin - Heidelberg - New York, 2003.

[2] B. Berkels, M. Burger, M. Droske, O. Nemitz, and M. Rumpf. Cartoon extraction based on anisotropic image classification, vision, modeling and visualization proceedings. In Vision, Modeling and Visualization. Springer, accepted. 

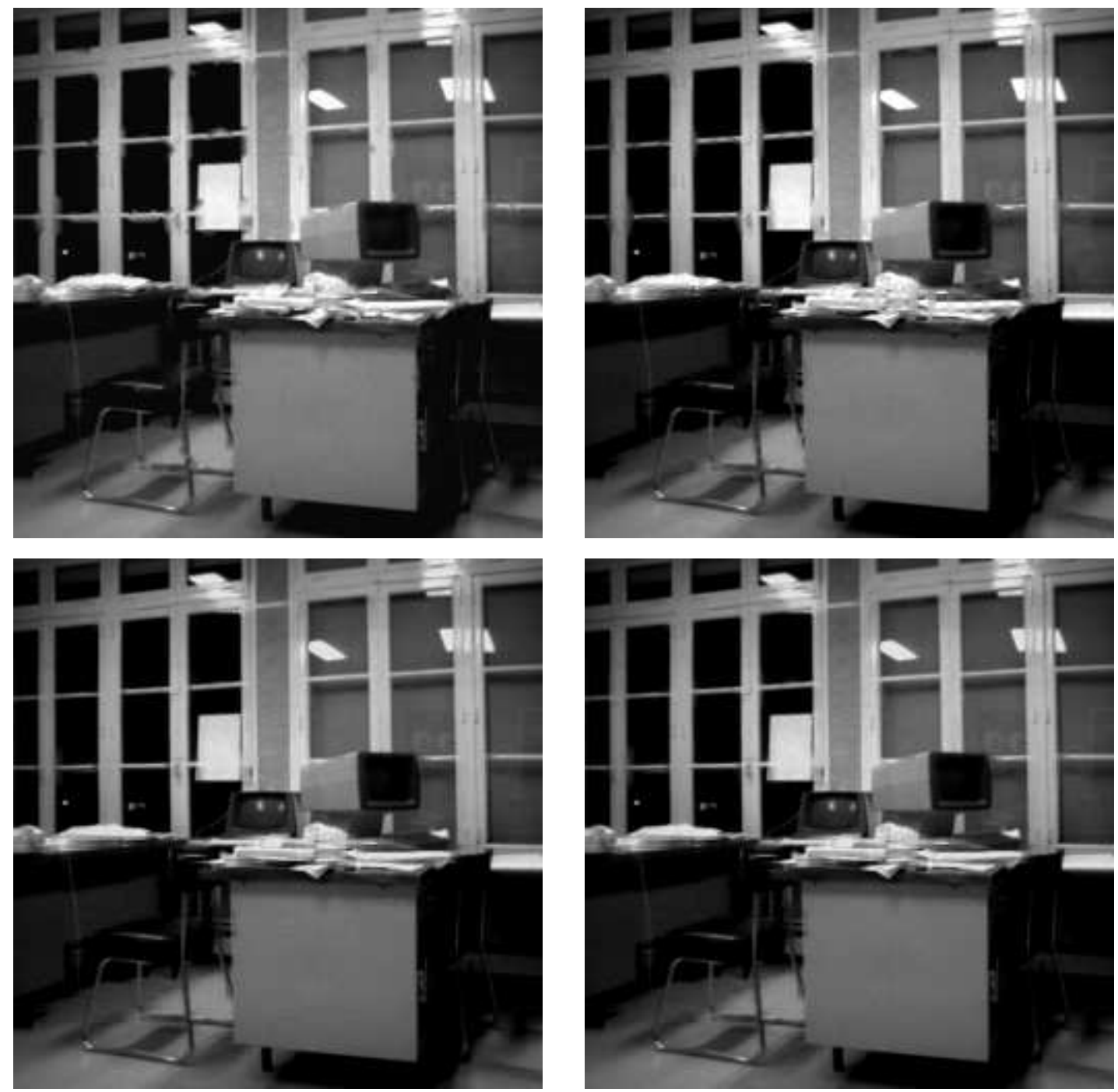

Figure 4: Interpolation of the image in Fig. 3. Top left: Cubic interpolation by the MATLAB procedure 'griddata' $\left(\mathrm{PSNR}=30.18, \mathrm{err}_{2}=7.89\right.$, $\left.\operatorname{err}_{1}=1.51\right)$. Top right: algorithm $\mathrm{A}$, $\left(\mathrm{PSNR}=31.61, \mathrm{err}_{2}=6.69, \mathrm{err}_{1}=1.36\right)$. Bottom left: algorithm $\mathrm{B},\left(\mathrm{PSNR}=34.08, \mathrm{err}_{2}=\right.$ $\left.5.03, \operatorname{err}_{1}=0.93\right)$. Bottom right: algorithm $\mathrm{C},\left(\mathrm{PSNR}=33.98, \operatorname{err}_{2}=5.09, \operatorname{err}_{1}=0.97\right)$. The images at the top contain artefacts, in particular at the left window side. The algorithms at the bottom show a better performance and do not introduce these artefacts.

[3] M. Bertalmio, G. Sapiro, V. Caselles, and C. Ballester. Image Inpainting. SIGGRAPH, $34: 417-424,2000$.

[4] M. Bertalmio, L. Vese, G. Sapiro, and S. Osher. Simultaneous structure and texture inpainting. IEEE Trans. Image Proc., 12(8):882-889, 2003.

[5] F. E. Browder and W. V. Petryshyn. The solution by iteration of nonlinear functional equations in Banach spaces. Bull. Amer. Math. Soc., 72(3):571-575, 1966. 

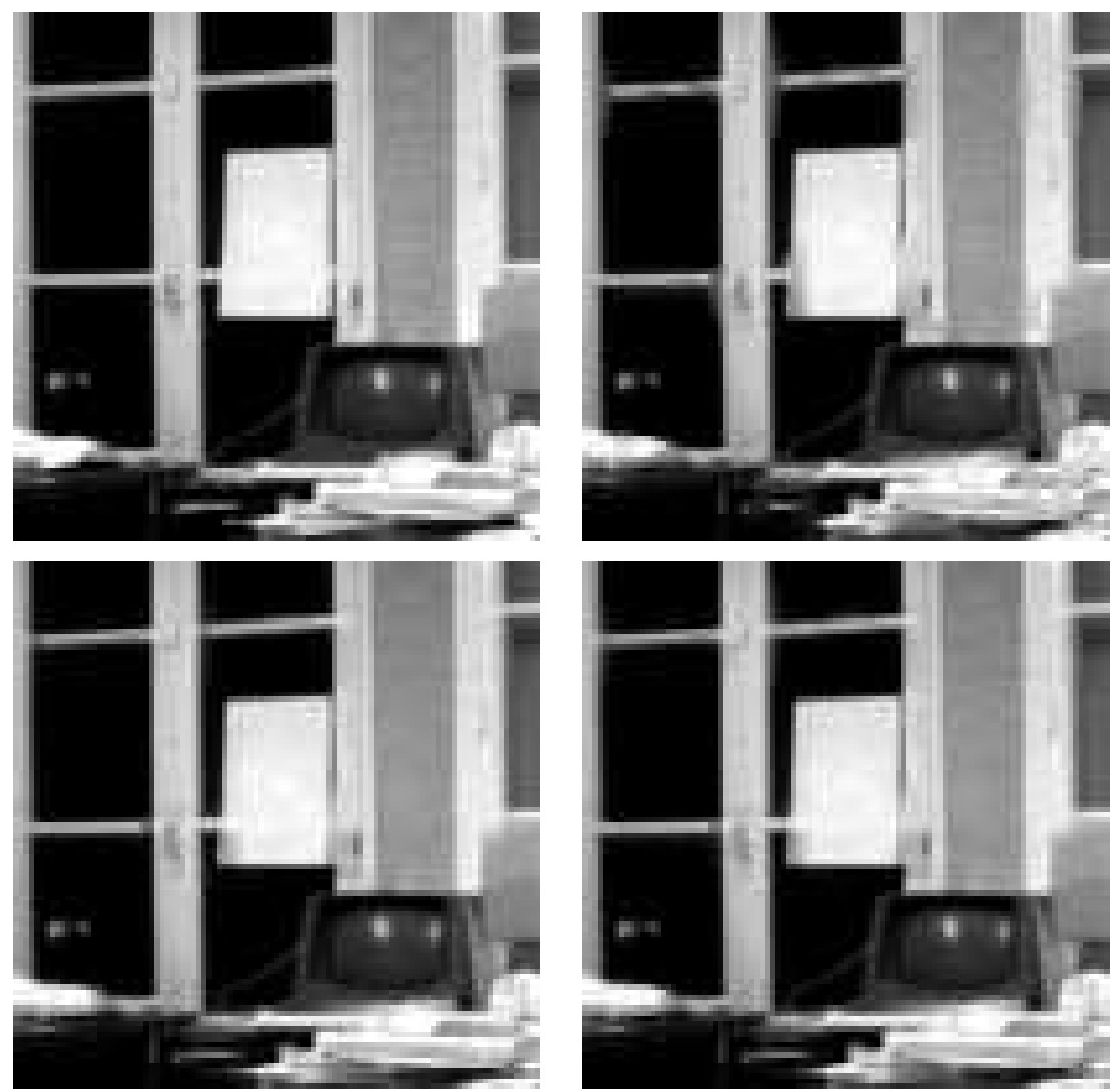

Figure 5: Details of the interpolated images of Fig. 4. Top left: original image. Top right: algorithm $\mathrm{A},\left(\mathrm{PSNR}=31.61\right.$, $\operatorname{err}_{2}=6.69$, $\left.\mathrm{err}_{1}=1.36\right)$. Bottom left: algorithm $\mathrm{B},\left(\mathrm{PSNR}=34.08, \mathrm{err}_{2}=5.03, \mathrm{err}_{1}=0.93\right)$. Bottom right: algorithm $\mathrm{C},(\mathrm{PSNR}=33.98$, $\operatorname{err}_{2}=5.09$, $\left.\operatorname{err}_{1}=0.97\right)$. In contrast to the top right image, the images at the bottom do not have high errors at the horizontal and vertical window lines.

[6] J.-F. Cai, R. H. Chan, and Z. Shen. A framelet-based image inpainting algorithm. Applied and Computational Harmonic Analysis, to appear.

[7] T. F. Chan, S. H. Kang, and J. Shen. Euler's Elastica and Curvature-Based Inpainting. SIAM Journal on Applied Mathematics, 63(2):564-592, 2002.

[8] P. L. Combettes and V. R. Wajs. Signal recovery by proximal forward-backward splitting. Multiscale Modeling and Simulation, 4:1168-1200, 2005. 

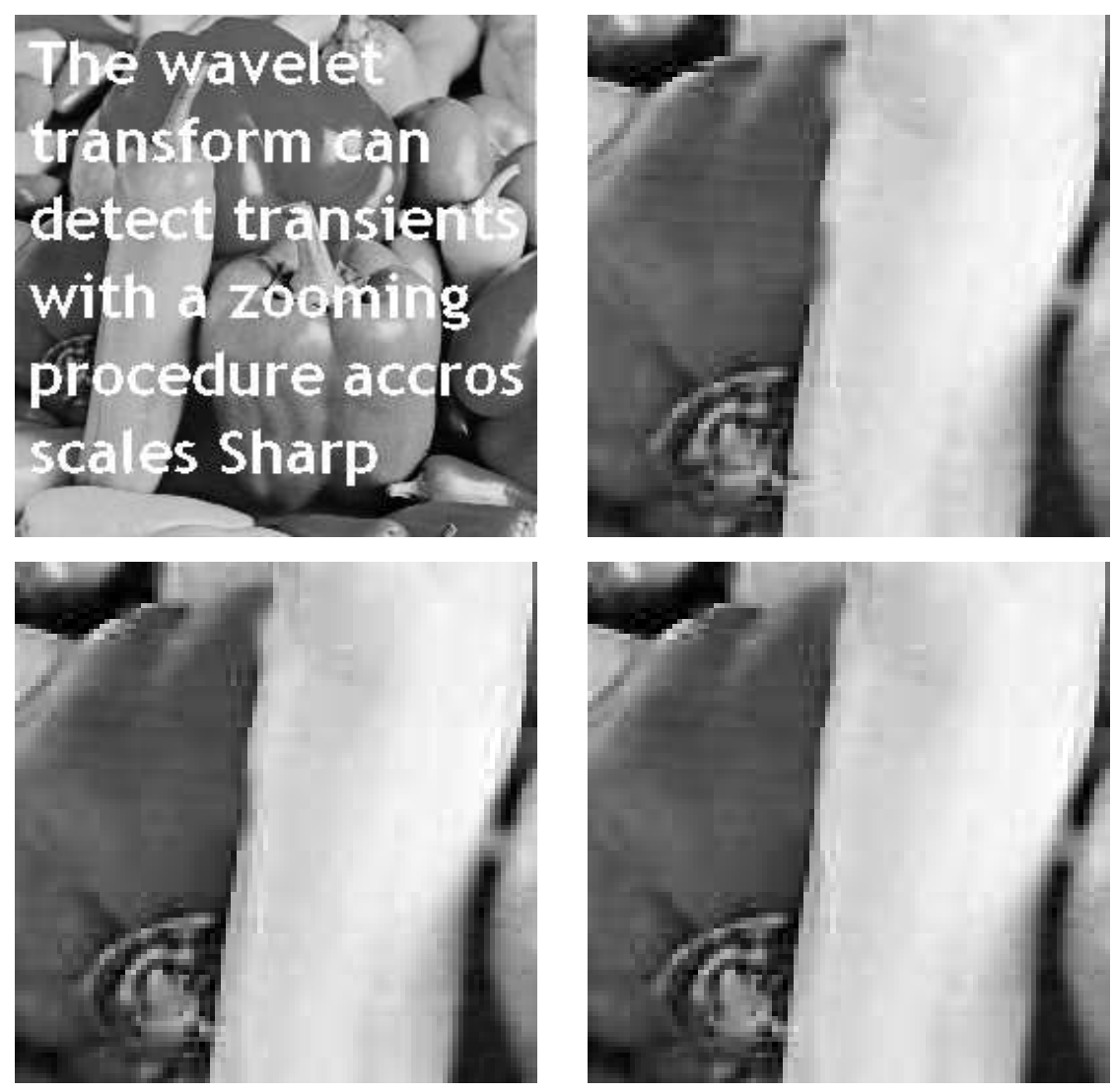

Figure 6: Interpolation results for the "peppers" image. Top left: degraded image. Top right: cubic interpolation. Bottom left: algorithm D. Bottom right: algorithm E. The algorithms D and $\mathrm{E}$ improve the quality at long edges.

[9] I. Daubechies, M. Defrise, and C. D. Mol. An iterative thresholding algorithm for linear inverse problems with a sparsity constraint. Communications in Pure and Applied Mathematics, 51:1413 - 1541, 2004.

[10] P. Davis. Circulant Matrices. J. Wiley and Sons, New York, 1979.

[11] S. Esedoglu and S. Osher. Decomposition of images by anisotropic Rudin-Osher-Fatemi model. Communications in Pure and Applied Mathematics, 57(12):1609-1626, 2004.

[12] M. J. Fadili and J.-L. Starck. Sparse representation and Bayesian image inpainting, volume 1 of Proc. SPARS'05. Rennes, France, 2005.

[13] D. Gabay, Applications of the method of multipliers to variational inequalities, In Augmented Lagrangian Methods: applications to the solution of boundary value problems, M. Fortin and R. Glowinski, eds., North Holland, Amsterdam, p. 299 - 331, 1983.

[14] I. Galić, J. Weickert, M. welk, A. Bruhn, A.Belyaev, and H.-P. Seidel, Towards PDEbased image compression. In Variational, Geometric, and Level Set Methods in Computer Vision, LNCS 3752, p. 37 - 48, Springer, Berlin, 2005. 
[15] W. Hintermüller and W. Kunisch. Total bounded variation regularization as a bilaterally constrained optimization problem. SIAM J. Appl. Math., 4(64):1311-1333, 2004.

[16] M. A. Krasnoselskii. Two observations about the method of successive approximations. Uspekhi Mat. Nauk, 10:123-127, 1955 (in Russian).

[17] P. L. Lions and B. Mercier. Splitting algorithms for the sum of two linear operators. SIAM J. Numer. Anal., 16:964-979, 1979.

[18] W. R. Mann. Mean value methods in iteration. Proc. Amer. Math. Soc., 4:506-510, 1953.

[19] G. J. Minty. Monotone (nonlinear) operators in Hilbert space. Duke Math. J., 29(3):341$346,1962$.

[20] G.B. Passty. Ergodic convergence to a zero of the sum of monotone operators in Hilbert space. J. Math. Anal. Appl., 72:383-390, 1979.

[21] L. I. Rudin, S. Osher, and E. Fatemi. Nonlinear total variation based noise removal algorithms. Physica D, 60:259-268, 1992.

[22] H. Schäfer. Über die Methode sukzessiver Approximationen. Jahresberichte Deutscher Math. Verein., 59:131-140, 1957 (in German).

[23] O. Scherzer and J. Weickert. Relations between regularization and diffusion filtering. Journal of Mathematical Imaging and Vision, 12:43-63, 2000.

[24] S. Setzer, G. Steidl, and T. Teuber. Restoration of images with rotated shapes. Numerical Algorithms, to appear.

[25] P. Tseng. Applications of a splitting algorithm to decomposition in convex programming and variational inequalities. SIAM J. Control Opt., 29(1):119-138, 1991.

[26] J. Weickert. Anisotropic Diffusion in Image Processing. Teubner, Stuttgart, 1998.

[27] M. Welk, G. Steidl, and J. Weickert. Locally analytic schemes: a link between diffusion filtering and wavelet shrinkage. Applied and Computational Harmonic Analysis, to appear.

[28] M. Elad, J.-L. Starck, P. Querre and D. L. Donoho. Simultaneous cartoon and texture image inpainting using morphological component analysis (MCA). Applied and Computational Harmonic Analysis, 19:340-358, 2005.

[29] M. Welk and J. Weickert. Tensor field interpolation with PDEs. In J. Weickert and H. Hagen, editors, Visualization and Processing of Tensor Fields, pages 315-325. Springer, Berlin, 2006. 